\title{
Characterization and transcriptomic analysis of a novel yellow-green leaf wucai (Brassica campestris L.) germplasm
}

Libing Nie ${ }^{1,2}$, Yushan Zheng ${ }^{1,2}$, Liting Zhang ${ }^{1,2}$, Ying $\mathrm{Wu}^{1,2}$, Shidong Zhu ${ }^{1,2,3}$, Jinfeng Hou ${ }^{1,2,3}$, Guohu Chen ${ }^{1,2}$, Xiaoyan Tang ${ }^{1,2}$, Chenggang Wang ${ }^{1,2,3^{*}}$ and Lingyun Yuan ${ }^{1,2,3^{*}}$

\begin{abstract}
Background: Leaf color mutants are the ideal materials to explore the pathways of chlorophyll (Chl) metabolism, chloroplast development, and photosynthesis system. In this study, a spontaneous yellow-green leaf wucai (Brassica campestris L.) mutant "WY16-13" was identified, which exhibited yellow-green leaf color during its entire growth period. However, current understanding of the molecular mechanism underlying Chl metabolism and chloroplast development of "WY16-13" is limited.

Results: Total Chl and carotenoid content in WY16-13 was reduced by 60.92 and $58.82 \%$, respectively, as compared with its wild type parental line W16-13. Electron microscopic investigation revealed fewer chloroplasts per cell and looser stroma lamellae in WY16-13 than in W16-13. A comparative transcriptome profiling was performed using leaves from the yellow-green leaf type (WY16-13) and normal green-leaf type (W16-13). A total of 54.12 million (M) (WY16-13) and 56.17 M (W16-13) reads were generated. A total of 40,578 genes were identified from the mapped libraries. We identified 3882 differentially expressed genes (DEGs) in WY16-13 compared with W16-13 (i.e., 1603 upregulated genes and 2279 downregulated genes). According to the Gene Ontology (GO) term and Kyoto Encyclopedia of Genes and Genomes (KEGG) pathway analyses, these DEGs are involved in porphyrin and $\mathrm{Chl}$ metabolism [i.e., chlorophyllase $(\mathrm{CLH})$, heme oxygenase $(\mathrm{HO})$, chlorophyll (ide) b reductase (NYC), and protochlorophyllide oxidoreductase $(P O R)$ genes], carbohydrate metabolism, photosynthesis, and carbon fixation in photosynthetic organisms. Moreover, deficiency in Chl biosynthetic intermediates in WY16-13 revealed that the formation of the yellow-green phenotype was related to the disorder of heme metabolism.
\end{abstract}

Conclusions: Our results provide valuable insights into $\mathrm{Chl}$ deficiency in the yellow-green leaf mutant and a bioinformatics resource for further functional identification of key allelic genes responsible for differences in Chl content.

Keywords: Transcriptome, Brassica campestris L. ssp. chinensis var. rosularis, Chlorophyll biosynthesis, Photosynthesis, Yellow-green leaf mutant

\footnotetext{
*Correspondence: ylyun99@163.com; cgwang@ahau.edu.cn

'College of Horticulture, Vegetable Genetics and Breeding Laboratory, Anhui

Agricultural University, 130 West Changjiang Road, Hefei 230036, Anhui,

China

Full list of author information is available at the end of the article
}

C C The Author(s). 2021 Open Access This article is licensed under a Creative Commons Attribution 4.0 International License, which permits use, sharing, adaptation, distribution and reproduction in any medium or format, as long as you give appropriate credit to the original author(s) and the source, provide a link to the Creative Commons licence, and indicate if changes were made. The images or other third party material in this article are included in the article's Creative Commons licence, unless indicated otherwise in a credit line to the material. If material is not included in the article's Creative Commons licence and your intended use is not permitted by statutory regulation or exceeds the permitted use, you will need to obtain permission directly from the copyright holder. To view a copy of this licence, visit http://creativecommons.org/licenses/by/4.0/. The Creative Commons Public Domain Dedication waiver (http://creativecommons.org/publicdomain/zero/1.0/) applies to the data made available in this article, unless otherwise stated in a credit line to the data. 


\section{Background}

Leaves are crucial organs that produce photosynthates for plant development and growth. Leaf color is mainly determined by pigment types and their relative concentrations. Chlorophyll (Chl) is the main pigment in leaves and are the primary photoreceptor pigments that capture light energy and drive electron transfer in the reaction center to form chemical energy and synthesize carbohydrates [1, 2]. In Arabidopsis thaliana, 27 genes involved in Chl metabolisms were identified, starting from glutamyl-tRNA to $\mathrm{Chl}$ a and $\mathrm{Chl} b$ [2].

Recently, mutants with disrupted Chl biosynthesis and degradation have been used to characterize steps associated with Chl metabolism in yellow-leaf plants such as rice [3-5], Arabidopsis thaliana [6], and pakchoi [7]. Numerous studies on other crops have been conducted to elucidate the molecular mechanisms of leaf color mutants, particularly leaf yellowing. Yellowgreen leaf mutants usually exhibit $\mathrm{Chl}$ deficiencies, shorter plant height, and retarded growth, resulting in decreased yield or even in plant death [8-10]. For instance, a yellow-green leaf phenotype is attributed to the disruption of the Chl synthase-encoding gene YGL1 or impaired chlorophyllide esterification, which results in lower Chl content and delayed chloroplast development [11, 12]. Virus-induced gene silencing of magnesium chelatase subunit D (CHLD) and I (CHLI) results in yellow phenotypes in peas with reduced $\mathrm{Chl}$ content and altered chloroplast function with abnormal chloroplast structure [13]. In rice, the pale-green leaf mutant pgl10 that is deficient in protochlorophyllide oxidoreductase (PORB) results in lower photosynthetic pigment content and grana lamellae in thylakoid compared to the wild-type [14]. Moreover, both external and internal factors could also influence Chl content such as light, salt, and osmotic stress due to alterations in gene expression or post-translational modification of proteins involved in Chl metabolism [15-17]. For example, a rice mutant deficient in fructose-1,6-bisphosphatase (FBFase) exhibits a yellow-green leaf phenotype and severe growth retardation [18]. In addition to alterations in Chl metabolism, the disruption of chloroplast function could also negatively affect $\mathrm{Chl}$ content and stability [15, 17]. The chloroplast plays a profound role in the biosynthesis of hormones, carbohydrates, and amino acids, as well as in energy metabolism. Peroxisomes, mitochondria, and chloroplast work cooperatively in plant energy metabolism pathways. Snowy cotyledon 3 (SCO3) localizes to peroxisomes, and its mutation in Arabidopsis leads to loss of Chl content and defective chloroplast function [19].

Wucai (Brassica campestris L. ssp. chinensis var. rosularis) belongs to non-heading Chinese cabbage family and is broadly cultivated in the Yangtze-Huai River basin in winter because of its cold-tolerant and high-quality features [20,21]. It has a variety of leaf colors and usual colors in the adult stage include dark green, green, outer dark green-inner green, outer green-inner light green, and outer light green-inner yellow. Currently, a novel wucai germplasm naturally occurs from a self-pollinated, stable, genetic high-generation inbred line "W16-13." It possesses a stable yellow-green leaf phenotype in the offspring at whole developmental stages and has been identified as "WY16-13" (Fig. 1). Its hybrid $F_{1}$ line, crossed with another green-leaf line, exhibits yellow-colored in inner leaves and green-colored in outer leaves and hybrid vigor. Therefore, "WY16-13" is an alternative and ideal yellow-green wucai germplasm to consider in future breeding programs and plantations. However, the molecular mechanism underlying its phenotype remains unclear.

In our study, the photosynthetic pigments, chloroplast ultrastructure, enzyme activity, and intermediate metabolites involved in Chl biosynthesis were assessed, and transcriptome level changes in WY16-13 and W16-13 were analyzed. Based on a combination of physiological and bioinformatics analyses, we identified DEGs related to Chl biosynthesis, and transcript levels of some key genes were evaluated to validate their involvement in leaf coloration. Our results illustrated the physiological and transcriptomic aspects of yellow-green leaf coloration in wucai and provide novel insights into the mechanism underlying $\mathrm{Chl}$ metabolism and chloroplast development.

\section{Results}

Phenotypic characterization of the WY16-13 mutant

Compared with W16-13, the leaves of WY16-13 exhibited yellow color during the entire growth period (Fig. 1). The color parameters $L^{\prime \prime}, a^{\prime \prime}$, and $b^{\prime \prime}$, which represented brightness, greenness, and yellowness, respectively, were significantly different between W16-13 and WY16-13. Compared with W16-13, L" and $b^{*}$ of WY16-13 were markedly higher, whereas a" was lower during the entire growth period (Fig. 2a-c). Additionally, measurement of the growth parameters showed that height and weight of WY16-13 were significantly lower than W16-13 throughout growth (Fig. 2d and e). Compared with W16-13, height of WY16-13 decreased by $23.09 \%$ at 35 d. Similarly, WY16-13 width decreased by $23.57 \%$ at $25 \mathrm{~d}$.

Assessment of chloroplast ultrastructure of the WY16-13 and W16-13 leaves was performed by the transmission electron microscopy (Fig. 3). Compared with the W16-13, WY16-13 exhibited fewer chloroplasts per cell and looser stroma lamellae. In addition, we observed a decrease in the number of starch 

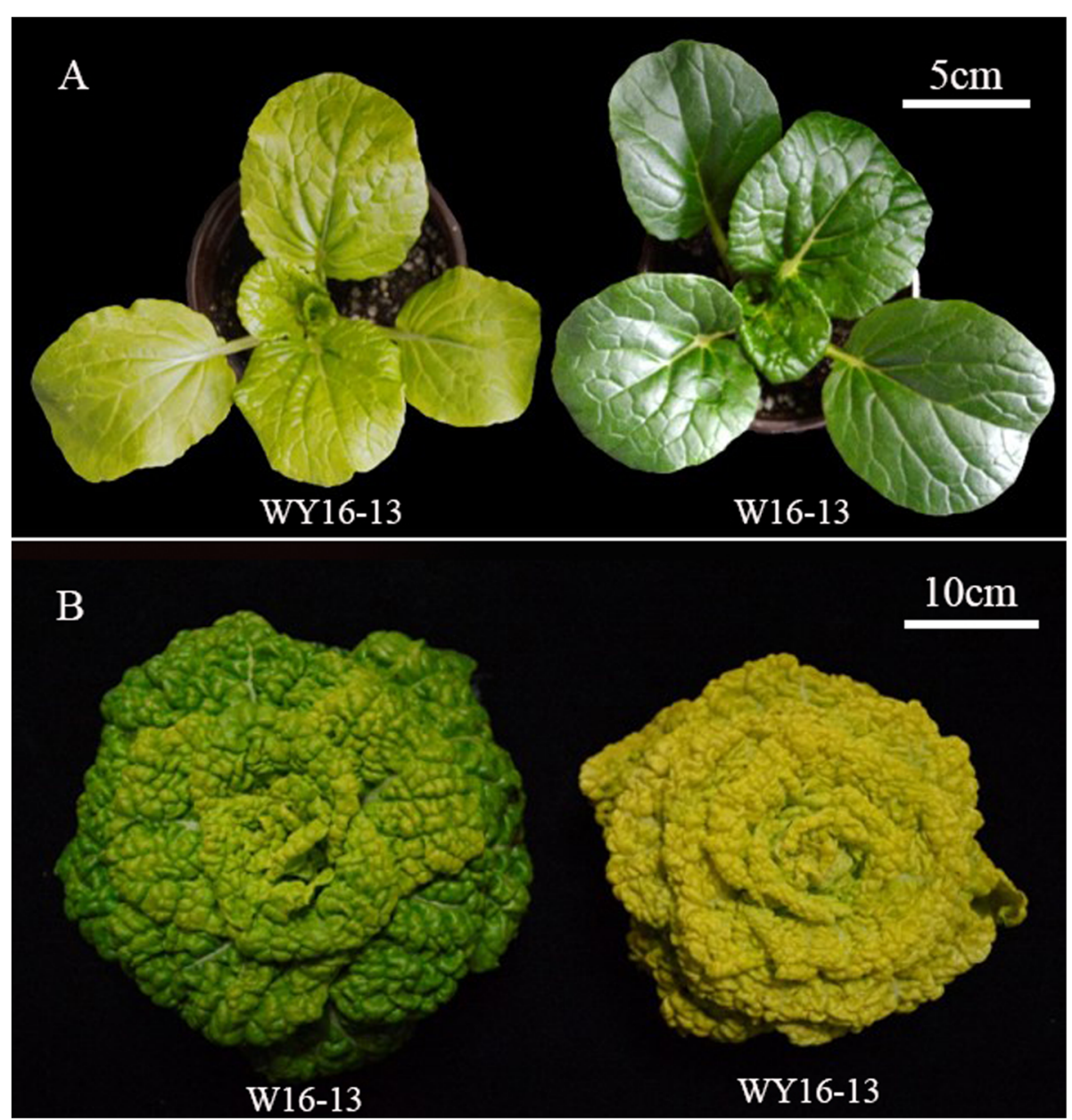

Fig. 1 Phenotype characterization of yellow-green leaf mutant WY16-13 and wild-type W16-13; WY16-13 and W16-13 at (a) seedling stage, Scale bar: $5 \mathrm{~cm}$; and (b) harvesting stage, Scale bar: $10 \mathrm{~cm}$

granules, an increase in the number of osmiophilic granules, and the lack of grana membranes in the chloroplasts of WY16-13 compared with the W16-13. These results indicated that the development of chloroplast was defective in WY16-13, which in turn disrupts plant photosynthesis.

\section{Chl metabolism analysis}

In the present study, the Chl and Carotenoid (Car) contents in W16-13 and WY16-13 leaves were measured (Table 1). The Chl and Car contents of the green leaves were significantly higher than those of the yellow leaves. $\mathrm{Chl} \mathrm{a}$, Chl b, and Car contents in WY16-13 significantly decreased by about $59.66,64.81$, and $58.82 \%$ relative to the leaves of the W16-13, respectively. These results suggest that the yellow mutant phenotype is probably due to reduced contents of photosynthetic pigments.

Furthermore, to investigate which biochemical step was disrupted and resulted in the yellow-leaf color phenotype, we assessed the levels of Chl biosynthesis intermediates in the W16-13 and WY16-13 leaves. Eight intermediate products that were related to Chl biosynthesis metabolic process were compared (Fig. 4). The results show that the Glutamate (Glu), 5-aminolevulinic acid (ALA), porphobilinogen (PBG), uroporphyrinogen III (Urogen III), coproporphyrinogen III (Coprogen III), Mg-protoporphyrin IX (Mg-Proto IX), protoporphyrin IX (Proto IX), and protochlorophyllide (Pchlide) contents of the leaves of WY16-13 plants were significantly lower than those of W16-13 plants. The levels of ALA and Proto IX in WY16-13 were only 46.24 and $23.27 \%$ of the W16-13, respectively.

\section{Sequencing and identification of expressed genes}

To explore molecular mechanism of the yellow-leaf phenotype of WY16-13, cDNA libraries of W16-13 and WY16-13 were constructed and based on three biological replicates (Table 2). The total number of pairedend reads in the three biological replicates of the W1613 was 56,516,916, 55,940,140, and 52,046,486, 


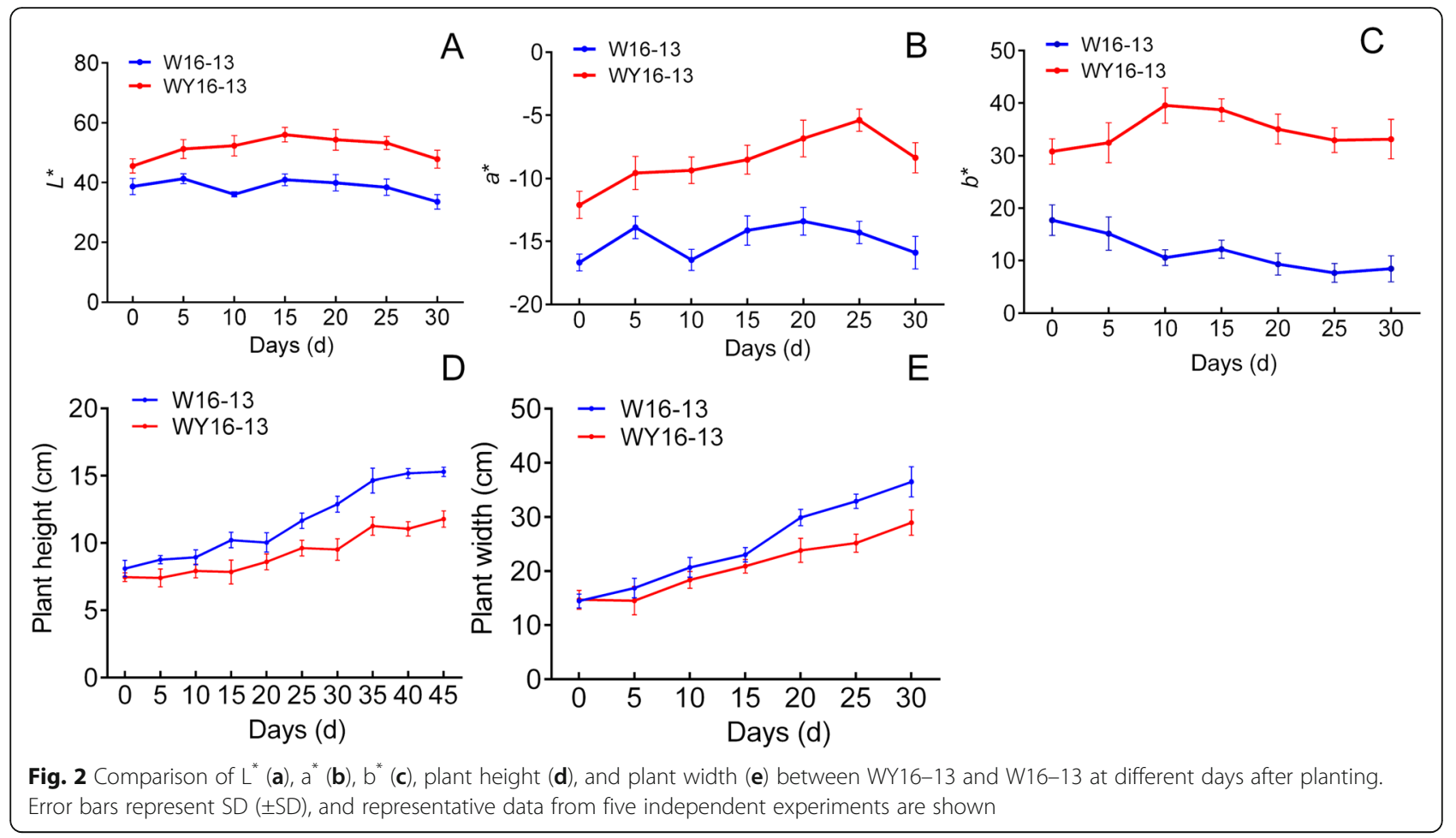

respectively, whereas that in WY16-13 was 51,448,202, $52,876,246$, and $54,528,540$. A total of $56.17 \mathrm{M}$ (W1613) and $54.12 \mathrm{M}$ (WY16-13) reads were generated. Moreover, the three samples of W16-13 and WY16-13 have strong correlation (Additional file 1: Figure S1 and Additional file 2: Figure S2). Clean reads were obtained by removing the adapters, low-quality, poly- $\mathrm{N}$, and empty reads, and the yield was about $7 \mathrm{G}$, with a GC percentage of about $47 \%$. The Q30 of the raw data ranged from 92.64 to $93.75 \%$, which indicated a high read confidence level. More than $88 \%$ (88.56-89.39\%) of the reads in the two transcriptome libraries mapped to the $B$. rapa reference genome. A total of 40,578 genes were identified from the mapped libraries.

\section{DEG identification}

Comparison of the yellow-green (WY16-13) leaves to the green (W16-13) leaves identified a total of 3882 DEGs, which included 1603 upregulated and 2279 downregulated genes (Fig. 5a and b). Hierarchical clustering of the DEGs was conducted to assess gene expression patterns and was calculated using the $\log _{10}$ RPKMs of the WY16-13 and W16-13 (Fig. 5c).

\section{GO and KEGG analyses of DEGs}

To explore the DEGs involved in yellow-green coloration, GO assignments were applied to classify the functions of the DEGs. A total of 3882 DEGs were divided into biological processes, cellular components, and molecular functions. Some DEGs were annotated with more than one GO term (Fig. 6a). In the biological process category, many genes belong to "release of seed from dormancy (GO:0048838)," "regulation of cell fate specification (GO:0042659)," "photosynthesis, light harvesting in photosystem I (GO:0009768)," and "response to abscisic acid (GO:0009737)." Some genes related to cellular component progress were involved in "photosystem II (GO:0009523)," "photosystem I (GO:0009522), " and "plastoglobuli (GO:0010287)." The molecular function group mainly included "chlorophyllase activity (GO: 0047746)," "phephytinase b activity (GO:0102293)," "hydroxyjasmonate sulfotransferase activity (GO: 0080131)," and "chlorophyll binding (GO:0016168)."

KEGG pathway analysis was conducted to categorize gene functions with an emphasis on biochemical pathways that were active in yellow-green and green leaves. A total of 1001 DEGs were annotated and assigned to processes such as cellular processes, environmental information processing, genetic information processing, metabolism, and biological systems (Additional file 1: Figure S1). Most of the DEGs enriched the functional subcategory of metabolism (Fig. 6b). The most enriched pathway was "carbohydrate metabolism," with 127 associated DEGs, followed by "amino acid metabolism" (94 DEGs) and "lipid metabolism" (84 DEGs). Many genes (111 DEGs) were belonged to the signal transduction group, which belongs to "environmental information processing." Moreover, 74 DEGs were assigned to 

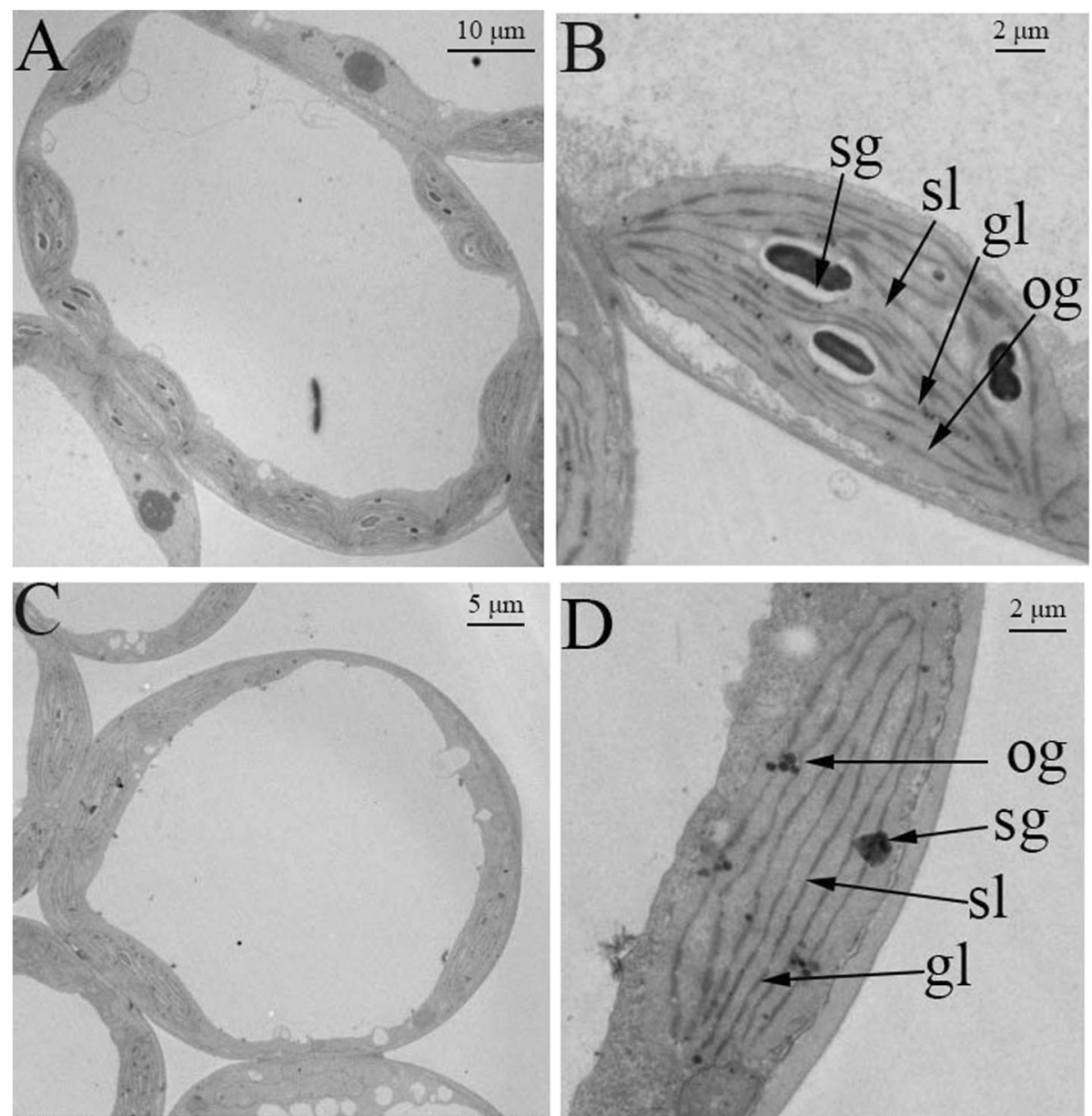

Fig. 3 Transmission electron micrograph of chloroplasts from mutant (WY16-13) and wild-type (W16-13) wucai. Structure of mesophyll cells in W16-13 (a) and WY16-13 (c). Structure of chloroplasts in W16-13 (b) and WY16-13 (d). sg: starch grains, sl: stroma lamellae, gl: grana lamellae, og: osmiophilic granules. a Bar: $10 \mu \mathrm{m}$; b Bar: $2 \mu \mathrm{m}$; c Bar: $5 \mu \mathrm{m}$; d Bar: $2 \mu \mathrm{m}$

"folding, sorting, and degradation" and 53 DEGs were assigned to "environmental adaptation." These results indicate that yellow-green and green leaves mainly differed in terms of metabolic processing. In addition, we focused on the analysis of DEGs related to chlorophyll metabolism. The results showed that Nine DEGs related to porphyrin and chlorophyll pathway (path: brp00860) were differentially expressed.

\section{Identification of DEGs related to $\mathrm{Chl}$ metabolism}

Based on the above annotations, the DEGs in the porphyrin and the Chl pathway (path: brp00860) were compared in detail between the WY16-13 and W16-13 transcriptomes (Additional file 4: Table S1). The results demonstrated that five genes in the porphyrin and $\mathrm{Chl}$ metabolism pathway were downregulated $(\mathrm{q}<0.05$, fold change $>2$ ), including two genes encoding $P O R B$ (LOC103861694 and LOC103867162), one gene encoding PORA (LOC103844881), one gene encoding CLH1 (LOC103872768), and a gene encoding HO1 (LOC103847911) (Fig. 7b). Four DEGs were upregulated, encoding CLH2 (LOC103848843 and LOC103839225), glutamate-tRNA ligase (LOC103854720), and NYC1 (LOC103833353) (Fig. 7b). These results indicated that

Table 1 Contents and relative ratio of photosynthetic pigments of leaves in W16-13 and WY16-13

\begin{tabular}{lllllll}
\hline Material & Total Chl content & Chl a content & Chl b content & Car content & Chl a/Chl b & Chl/Car \\
\hline W16-13 & $1.74 \pm 0.12^{* *}$ & $1.19 \pm 0.06^{* *}$ & $0.54 \pm 0.06^{* *}$ & $0.17 \pm 0.01^{* *}$ & $2.22 \pm 0.16$ & $9.94 \pm 0.72$ \\
WY16-13 & $0.68 \pm 0.01$ & $0.48 \pm 0.01$ & $0.19 \pm 0.01$ & $0.07 \pm 0.01$ & $2.47 \pm 0.09$ & $9.05 \pm 1.19$
\end{tabular}

Error bars indicate means \pm SD based on three independent experiments. Significant differences were determined using the Student's $t$ test in WY16-13 compared with W16-13 $(* * 0<0.01)$ 


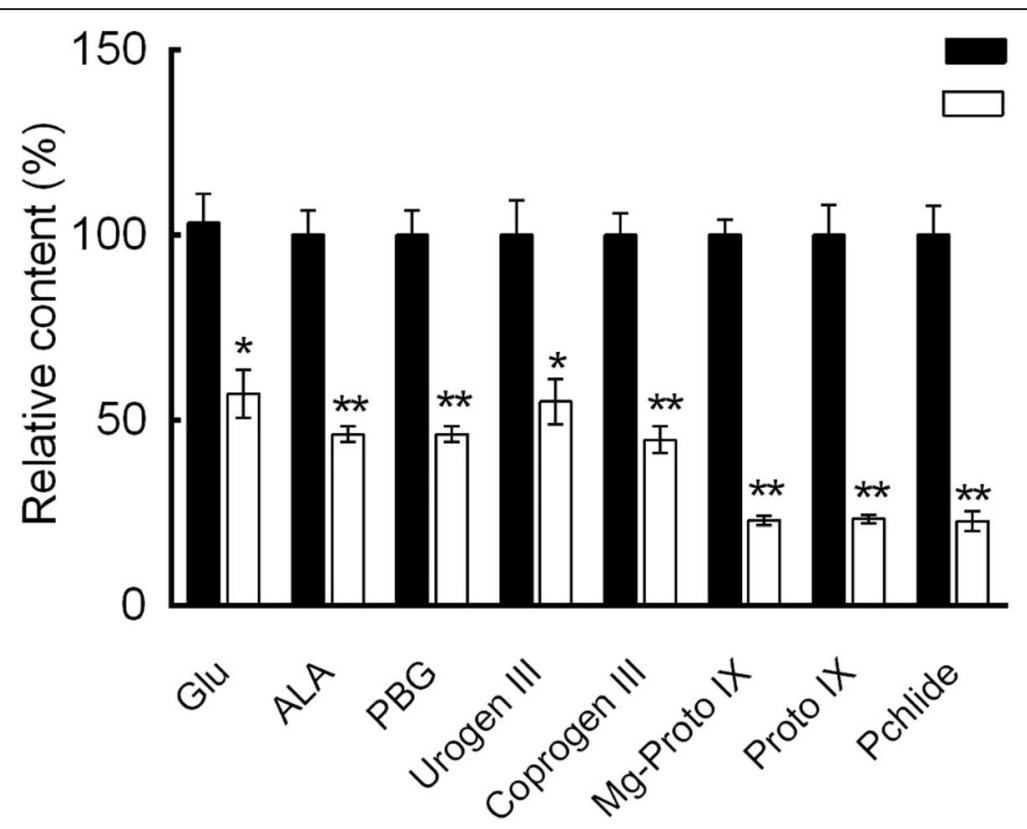

Fig. 4 Comparison of the relative contents of chlorophyll precursors. Three individuals were measured for each chlorophyll precursor. Error bars indicate means \pm SD based on three independent experiments. Significant differences were determined using the Student's $t$ test in WY16-13 compared with W16-13( ( $^{2}<0.05$, $\left.^{* *} p<0.01\right)$. Glu, Glutamate; ALA, 5-aminolevulinic acid; PBG, porphobilinogen; Urogen III, uroporphyrinogen III; Coprogen III, coproporphyrinogen III; Proto IX, protoporphyrin IX; Mg-Proto IX, Mg-protoporphyrin IX; Pchlide, protochlorophyllide

green and yellow leaves largely differed in metabolic activities.

\section{Photosynthesis analysis}

In the photosynthesis pathway (brp00195 and brp00196), a total of 40 DEGs encoding core proteins of Photosystem II (PSII), Photosystem I (PSI), the light-harvesting chlorophyll protein complex (LHC), and the photosynthetic electron transport were all downregulated in the WY16-13 mutant (Fig. 8). In PSII, the DEGs included oxygen-evolving enhancer protein 1 (Psb O), oxygen-evolving enhancer protein 2 (Psb P), Psb W protein (Psb W), psbY protein (Psb $\mathrm{Y}$ ), and $10 \mathrm{kDa}$ polypeptide (Psb R). In PSI, the DEGs included subunit II (Psa D), subunit IV A (Psa E), subunit III (Psa F), subunit V (Psa G), subunit VI (Psa $\mathrm{H}$ ), and subunit Psa K, Psa O, and Psa N. In photosynthetic electron transport, the DEGs included ferredoxin (Pet F) and plastocyanin (Pet E). In addition, 15 genes encoding the chlorophyll a/b-binding protein were suppressed in the WY16-13 mutant (Additional file 5: Table S2). These results suggest that suppression of these photosynthetic genes was responsible for defects in chloroplast development in the WY16-13 mutant.

The Chl a fluorescence transient reflects the effect of PSII after quantitatively analyzing changes in the OJIP curve (Fig. 9a). The chlorophyll fluorescence parameters of "WY16-13" and "W16-13" are shown in Fig. 9b and Table 3. The results show that the levels of $\mathrm{F}_{0}, \mathrm{Fm}, \mathrm{ABS} / \mathrm{RC}$, and $\mathrm{DI}_{\mathrm{o}} / \mathrm{RC}$ of WY16-13 were significantly lower than W16-13. Compared with W16-13, $\mathrm{F}_{0}, \mathrm{~F}_{\mathrm{m}}, \mathrm{ABS} / \mathrm{RC}$, and $\mathrm{DI}_{\mathrm{o}} / \mathrm{RC}$ levels in WY16-13 decreased by 29.36, 21.88, 14.81, and $25.40 \%$, respectively. Conversely, $\mathrm{F}_{\mathrm{v}} / \mathrm{F}_{\mathrm{m}}, \mathrm{PI}_{\mathrm{abs}}$, and $\mathrm{PI}_{\text {total }}$ levels in WY16-13 were significantly higher than W16-13, which increased by $1.86,37.12$, and $154.36 \%$, respectively.

Table 2 The quality of the transcriptome of WY16-13 and W16-13 leaves

\begin{tabular}{llllllll}
\hline Sample & raw_reads & clean_reads & clean_bases & Q30 & GC & Total number of reads & Total number of mapped reads \\
\hline W16_13a & $57.87 \mathrm{M}$ & $56.52 \mathrm{M}$ & $7.47 \mathrm{G}$ & $93.37 \%$ & $48.47 \%$ & $56,516,916$ & $50,520,317(89.39 \%)$ \\
W16_13b & $57.33 \mathrm{M}$ & $55.94 \mathrm{M}$ & $7.38 \mathrm{G}$ & $93.65 \%$ & $48.27 \%$ & $55,940,140$ & $49,750,259(88.93 \%)$ \\
W16_13C & $53.32 \mathrm{M}$ & $52.05 \mathrm{M}$ & $6.93 \mathrm{G}$ & $93.37 \%$ & $48.39 \%$ & $52,046,486$ & $46,372,520(89.10 \%)$ \\
WY16_13a & $52.65 \mathrm{M}$ & $51.45 \mathrm{M}$ & $6.88 \mathrm{G}$ & $93.75 \%$ & $47.92 \%$ & $51,448,202$ & $45,657,240(88.74 \%)$ \\
WY16_13b & $53.97 \mathrm{M}$ & $52.88 \mathrm{M}$ & $7.09 \mathrm{G}$ & $93.20 \%$ & $47.82 \%$ & $52,876,246$ & $46,827,653(88.56 \%)$ \\
WY16_13C & $55.74 \mathrm{M}$ & $54.53 \mathrm{M}$ & $7.26 \mathrm{G}$ & $92.64 \%$ & $48.04 \%$ & $54,528,540$ & $48,650,218(89.22 \%)$ \\
\hline
\end{tabular}



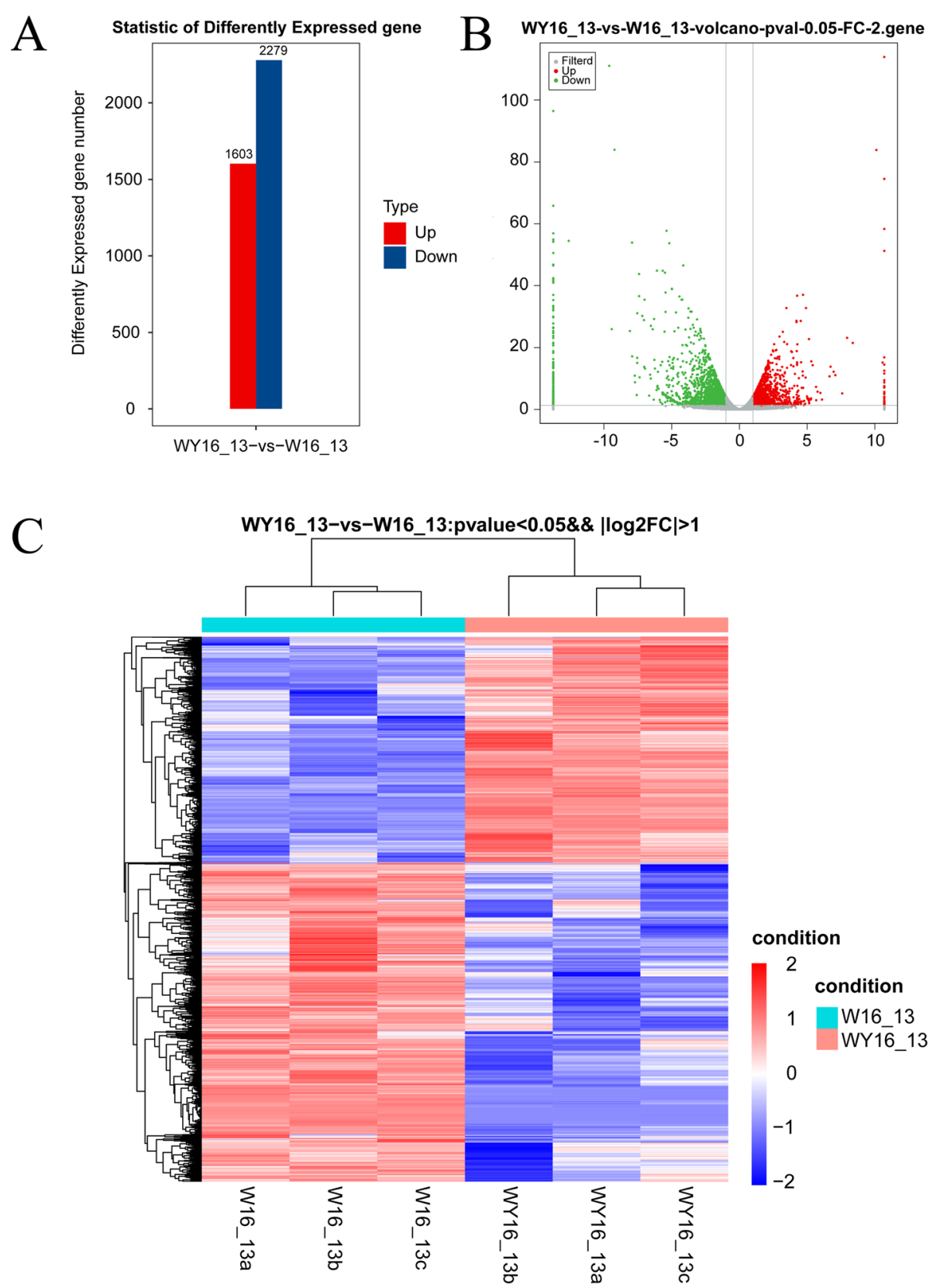

Fig. 5 Transcriptome analysis of DEGs in WY16-13 and W16-13. Comparison of DEGs in WY16-13 and W16-13 (a). Volcano plot showing the DEGs between two different libraries (b). The threshold $q<0.05$ was used to determine the significance of DEGs. Red and green dots represent up- and downregulated genes, respectively, and gray dots indicate transcripts that did not significantly change in the WY16-13 library compared to W16-13. Hierarchical clustering of all of the DEGs was based on the $\log _{10}$ RPKM values (c). The color spectrum from blue to red represents gene expression intensity, ranging from low to high, respectively

\section{Quantitative real-time PCR (qRT-PCR) analysis}

To validate the reliability of the DEG expression, 20 DEGs were randomly selected for qRT-PCR analysis. The expression patterns revealed by qRT-PCR analysis were similar to those obtained by RNA-Seq for the same genes (Fig. 10). These findings indicate that the RNAseq results of the present study are reliable for all kinds of analysis.

\section{Discussion}

Chl deficiency and abnormal chloroplast development can lead to the plant leaf yellowing phenotype, which is closely related to photosynthesis. WY16-13 is a spontaneous yellow-green leaf color mutant of the cultivar W16-13 in wucai that presents a yellow-green leaf phenotype during the entire growth period. In this study, we conducted comprehensive biochemical analysis and 
A

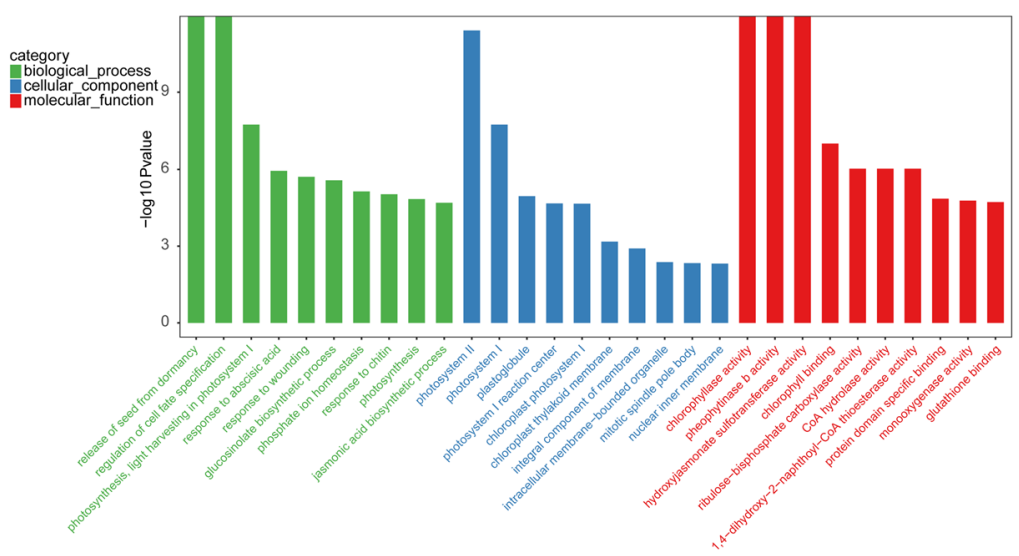

B

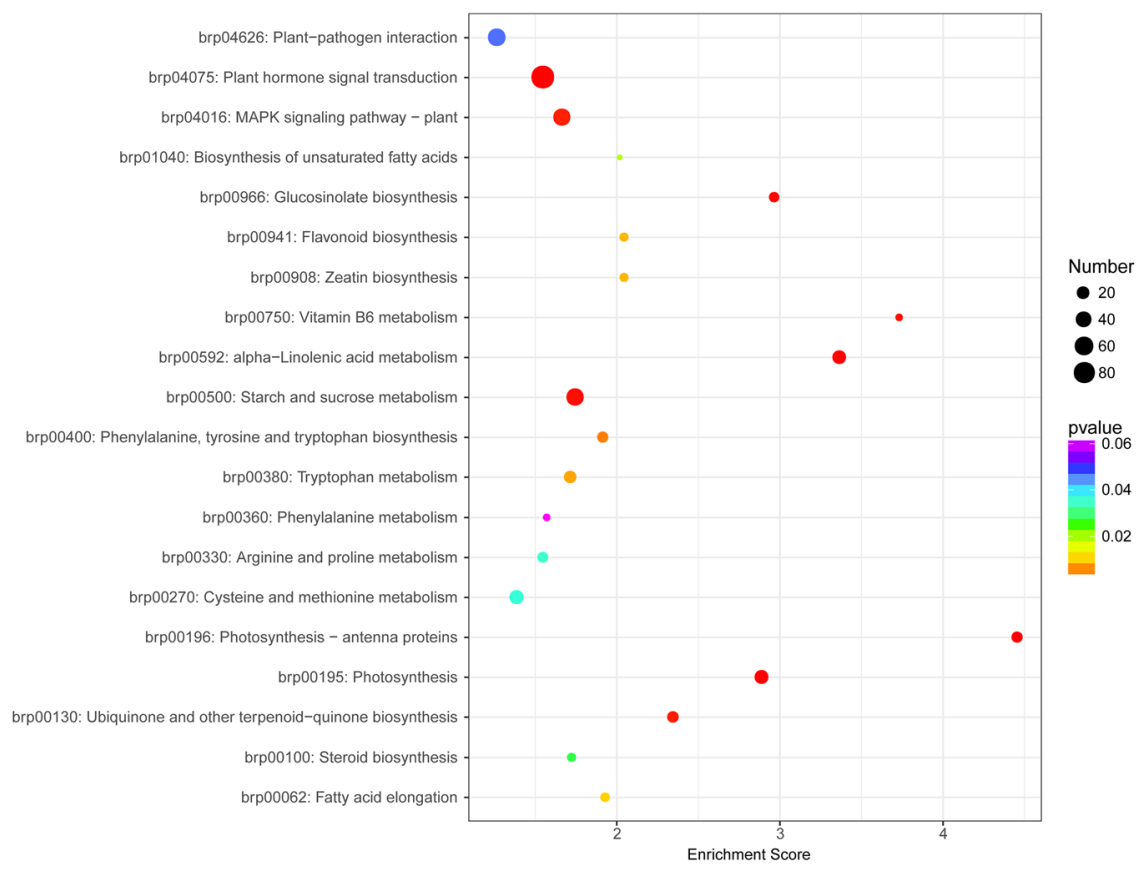

Fig. 6 GO and KEGG pathway enrichment analyses of DEGs between WY16-13 and W16-13. GO enrichment analysis with the 30 most enriched $\mathrm{GO}$ terms in the three categories shown (a). KEGG enrichment analysis with the 20 most enriched KEGG terms shown (b). High and low $p$-values are represented by blue and red, respectively

transcriptome profiling of WY16-13 and W16-13 to obtain insights into leaf color variations at the transcriptional level and complex biological processes.

Leaves are the economically significant and utilized parts of vegetable plants and are also the primary sites of photosynthesis. Changes in leaf color are mainly determined by complex biological processes. Chl is the main pigment that harvests solar energy in leaf tissues. In this study, comparative analysis of photosynthetic pigment content in the leaves of WY16-13 and W16-13 revealed that the Chl and Car contents in WY16-13 were significantly lower than W16-13. We hypothesize that the yellow-green phenotype of WY16-13 leaves is caused by a deficiency in Chl, which has also been reported in Arabidopsis [6], soybean [22], rice [23], and cucumber [24].

Chl metabolism is determined by complex biological processes, and blockage of any step in this process can lead to a decrease in Chl content, which in turn results in a change in leaf color. Comparative transcriptome profiling of WY16-13 and W16-13 revealed that three genes encoding POR were downregulated (Fig. 7). POR is a key enzyme for Chl biosynthesis that catalyzes the photoreduction of protochlorophyllide to chlorophyllide. It has been reported that mutations in the $P O R B$ gene lead to a yellow/white leaf variegation phenotype in rice [5]. In addition, in Arabidopsis, the transcriptional activity of PORB and PORC in the yellow tissues of 


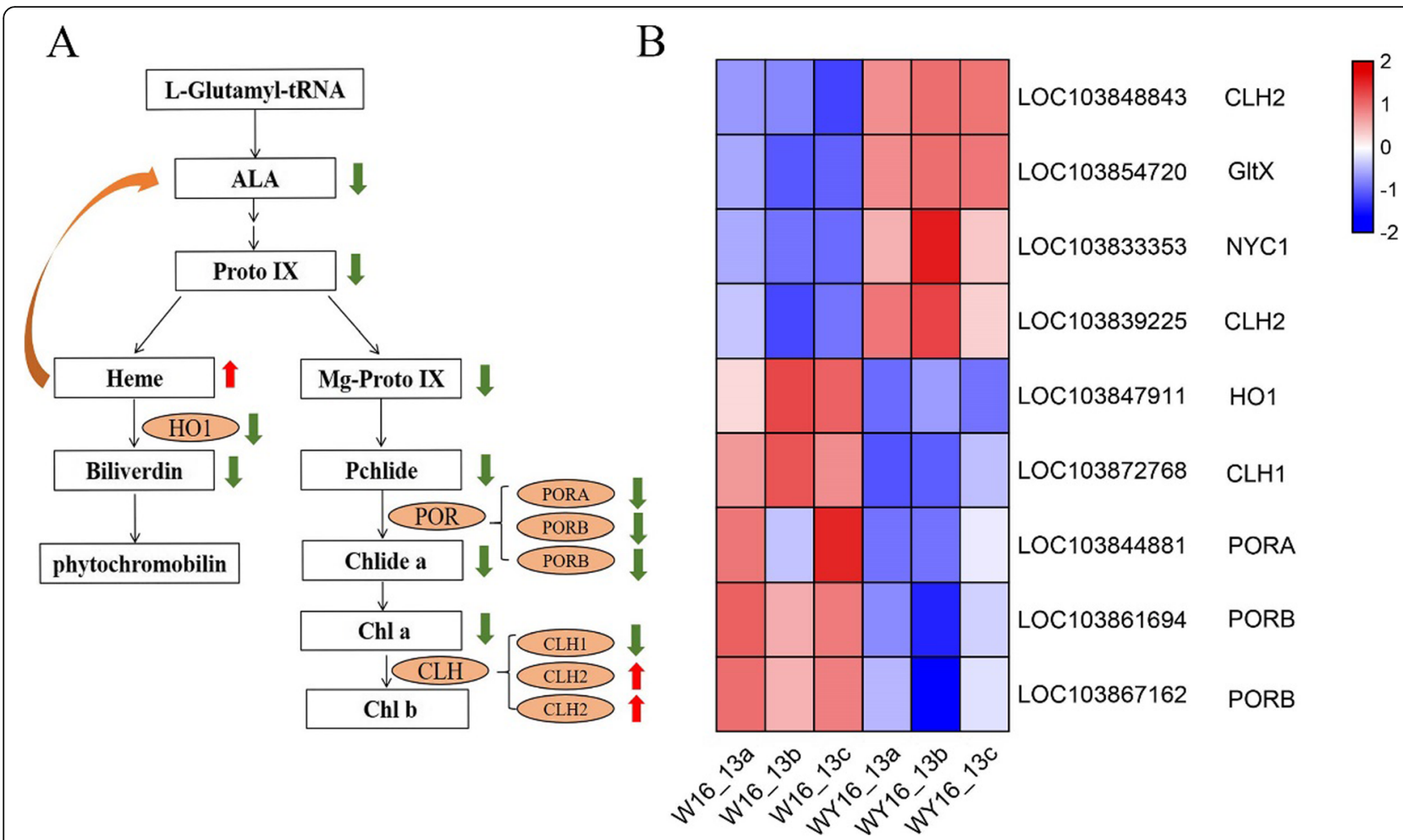

Fig. 7 DEGs involved in the chlorophyll biosynthesis pathway. Chlorophyll biosynthesis pathway (a); upregulated genes are marked by red arrows and downregulated genes by green arrows. Expression profile clustering of the chlorophyll biosynthesis pathway (b). Expression ratios are based on $\log _{2}$ fragments per kilobase of transcript per million mapped reads (FPKM) values, where each vertical column represents a sample (W16_13a, W16_13b, and W16_13c; WY16_13a, WY16_13b, and WY16_13c), and each horizontal row represents a single gene. CLH, chlorophyllase; POR, protochlorophyllide oxidoreductase; $\mathrm{HO}$, heme oxygenase; NYC, probable chlorophyll (ide) b reductase

variegated mutants is either absent or very low [25]. These results suggest that inhibition of POR in the yellow leaf mutant reduces $\mathrm{Chl}$ content and leads to the yellow leaf phenotype. Moreover, two genes encoding CLH and one gene encoding NYC were upregulated in yellow leaf plants. Chlorophyllase is considered to be a rate-limiting enzyme that catalyzes the hydrolysis of chlorophyll to form chlorophyllide [26]. NYC1 protein is a chlorophyll b reductase that is highly similar to shortchain dehydrogenases/reductases [27]. Mutation of the $N Y C 1$ gene leads to a stay-green phenotype during senescence in rice [28]. A previous study on Cymbidium orchids has suggested that the yellow-green leaf phenotype may be related to the upregulated expression of CLH [29]. Our results indicate that the upregulated expression of the CLH and NYC1 genes in WY16-13 may accelerate Chl breakdown, lead to yellowing of plant leaves.

In addition, the HO1 gene in WY16-13, which encodes heme oxygenase, was significantly ( $\log _{2}$ FoldChange $=-5.01$ ) downregulated. Heme oxygenase is an important rate-limiting enzyme of heme metabolism and can cleave protoheme to form biliverdin, which in turn releases $\mathrm{Fe}^{2+}$ and carbon monoxide [30]. Disruption of heme metabolism in plants may inhibit Chl biosynthesis and cause leaf chlorosis [31]. In the rice yellowing mutant $y l c 2$, a fragment deletion of the osHO2 by map cloning resulted in the yellowing of young leaves [32]. By comparing and analyzing the content of Chl metabolism intermediate products, we found that the content of eight intermediate products in WY16-13 was significantly lower than W16-13 (Fig. 4). The Chl biosynthesis-hindered site of WY16-13 does not occur in the process from ALA to Chl. Therefore, the lack of Chl in WY16-13 may be caused by a disruption of heme metabolism. Excessive heme accumulation inhibits the activity of glutamyl-tRNA and the synthesis of ALA, thereby reducing the overall rate of tetrapyrrole biosynthesis, ultimately leading to a decrease in Chl synthesis. The rice $y g l 2$ mutant has an insertion mutation in the heme oxygenase 1, which leads to a significant reduction of $\mathrm{HO} 1$ expression level, resulting in a yellow-green leaf phenotype [33]. Similar results have been found in hy1 mutants of $A$. thaliana [34].

Chloroplasts are cytoplasmic organelles in eukaryotic cells that consist of a chloroplast membrane, thylakoid, and matrix and are the sites of photosynthesis [35]. Chloroplasts contain a highly folded thylakoid 


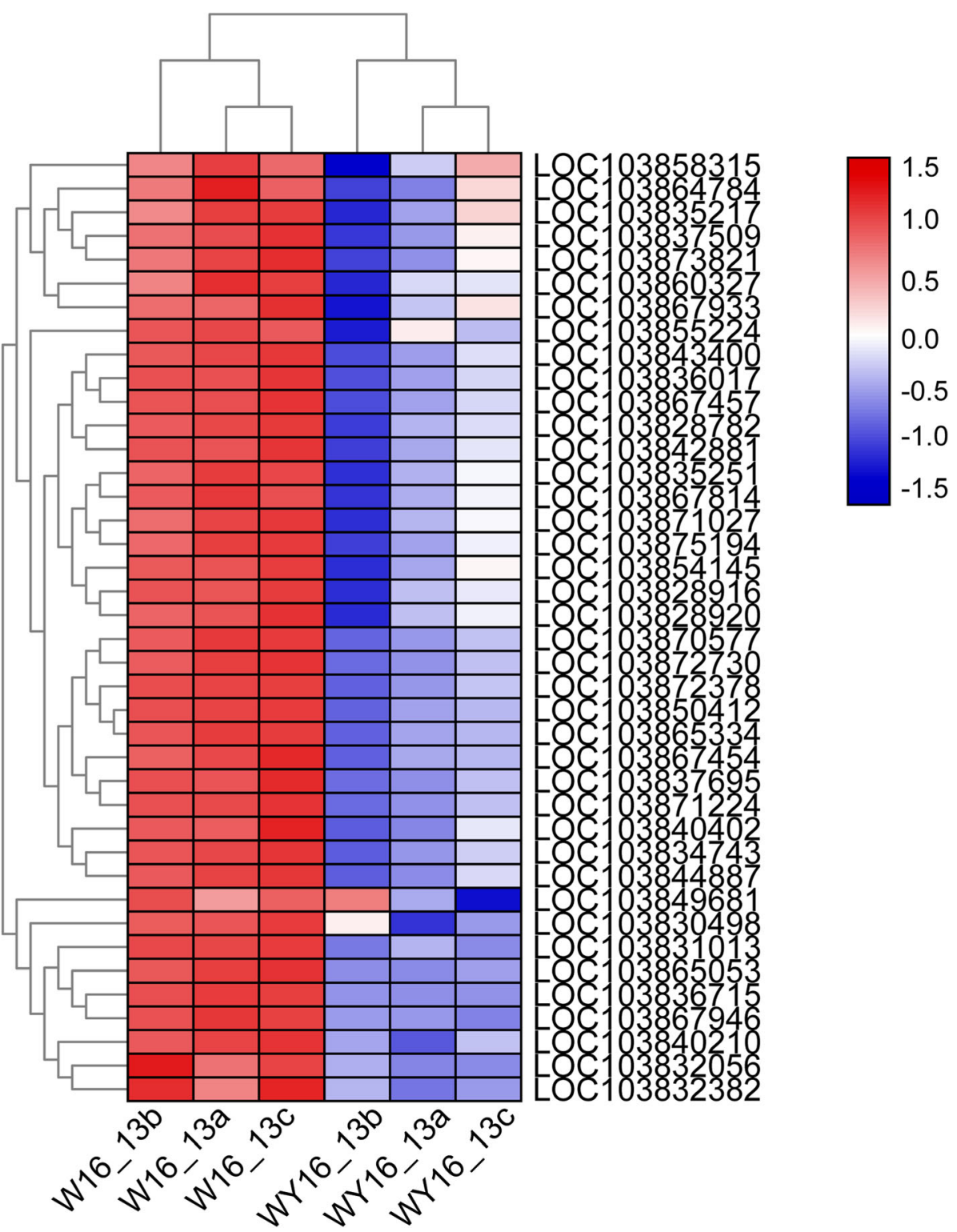

Fig. 8 Heat map of the photosynthesis- and photosynthesis antenna protein-related DEGs in the leaves of W16_13a, WY16_13b, and WY16_13c and WY16_13a, WY16_13b, and WY16_13c. Expression ratios are based on $\log _{2}$ FPKM values. Red and blue represent high or low expression levels, respectively, than those shown in white

membrane system that includes the photosystems (PSII and PSI) and the cellular structure responsible for the generation of proton motility [36]. Metabolism and accumulation of $\mathrm{Chl}$ are inseparable from the normal development of chloroplasts. Leaf color yellowing may be related to the development of chloroplasts. Proteins plays an important role in the process of chloroplast development and differentiation [37]. Change in the coding region, synthesis, transportation, recognition, and binding of chloroplast proteins will directly or indirectly affect the synthesis and accumulation of Chl, which in turn results in leaf color variations [38]. Based on transcriptome data, we identified 40 DEGs that are related to photosynthesis between WY16-13 and W1613. The DEGs are enriched in the photosynthetic pathway of plants such as (PSI, PSII, light harvesting complexes, cytochrome b6/f, and ATP synthase) and all downregulated (Fig. 8). These results are concordant to the findings of our previous analysis of the ultrastructure of chloroplasts, indicating that yellowing of leaves is largely affected by abnormal chloroplast development.

In higher plants, early light-induced proteins (ELIPs) are nuclear-encoded light stress-induced proteins in the thylakoid membrane system, where these are proposed to 

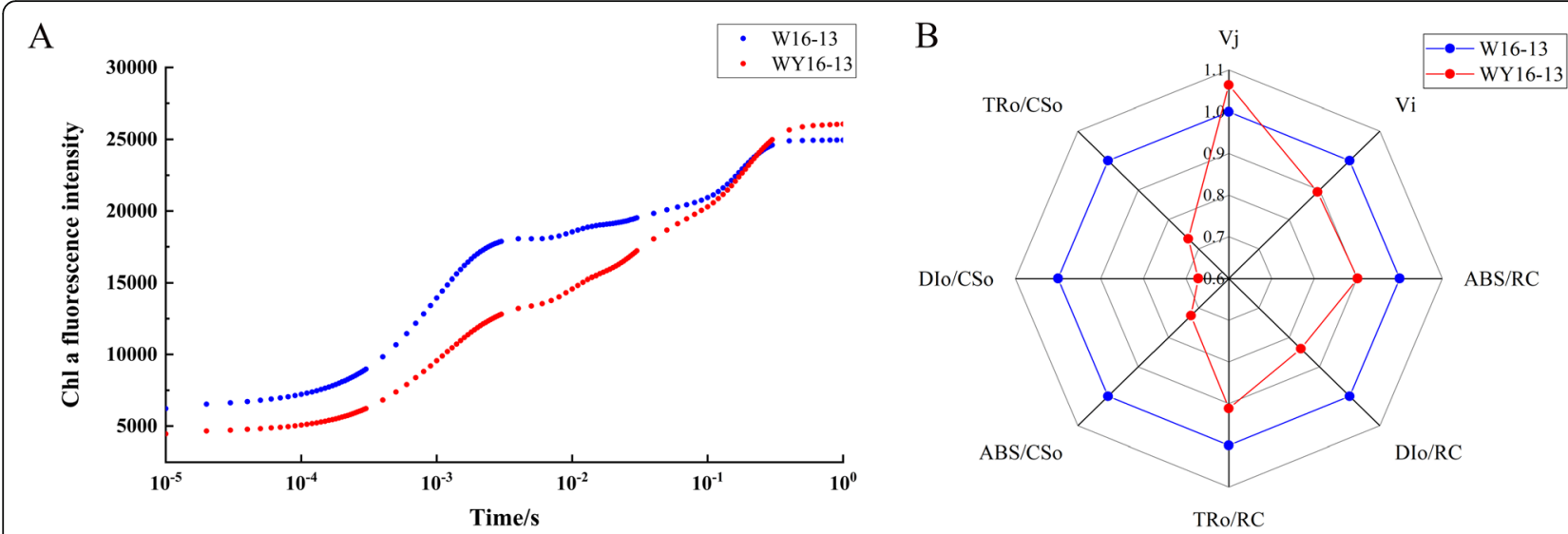

Fig. 9 Fast Chl a fluorescence transient (OJIP) plotted on logarithmic time scale (0.00001-1 s) measured in WY16-13 and W16-13 (a), and Chl fluorescence kinetic parameters of WY16-13 and W16-13 (b)

function in photoprotection [39-41]. The accumulation of ELIP transcripts and proteins is related to the degree of photoinactivation and photodamage of the PSII reaction center [42]. In WY16-13, the accumulation of ELIP1 mRNA was 2.06-fold higher compared with W16-13, resulting in leaf color variations (Additional file 6: Table S3). Previous studies on Arabidopsis have suggested that the activity of glutamyl tRNA reductase, CHLH, and CHLI is reduced in ELIP2 gene overexpression plants, resulting in a decrease in plant Chl content. Therefore, changes in the expression of genes related to carbon metabolism and photosynthesis in mutants may lead to abnormal chloroplast development and reduced $\mathrm{Chl}$ content. In addition, the Golden 2-like (GLK) families have been reported to be involved in the positive regulation of chloroplast development [43, 44]. In this study, we identified three GLK transcription factors, including two GLK1 genes (LOC103842594 and LOC103828162) and one GLK2 gene (LOC103827922) (Additional file 6: Table S3). It is worth noting that the GLK1 gene was upregulated 106.09-fold in the mutant. The GLK transcription factors encode transcriptional activators that promote the expression of nuclear-encoded photosynthetic genes that are required for Chl biosynthesis and light harvesting functions [45]. In Arabidopsis, the glk1-glk2 double mutants exhibit a pale green phenotype and its chloroplasts lack thylakoid membranes and grana [46, 47]. These results further indicate that the expression levels of GLKs are closely related to chloroplast development and Chl biosynthesis.

\section{Conclusions}

In summary, the physiological characteristics of the yellowgreen leaf mutant "WY16-13" and normal green color cultivar "W16-13" were analyzed. The lower Chl contents and abnormal ultrastructure of chloroplasts in the leaves of WY16-13 suggested that Chl biosynthesis was partially inhibited. We performed transcriptome analysis by RNAseq to elucidate the molecular mechanism underlying $\mathrm{Chl}$ metabolism and chloroplast development in the yellowgreen leaf mutant. We identified nine DEGs that were related to the porphyrin and $\mathrm{Chl}$ metabolism. Among these, Chl biosynthesis genes, including LOC103844881, LOC103861694, and LOC103867162 were downregulated in the mutant, while Chl degradation genes, including LOC103848843, LOC103833353, and LOC103839225, were upregulated. In addition, the downregulated $\mathrm{HOl}$ gene,

Table 3 Chl fluorescence kinetic parameters of WY16-13 and W16-13

\begin{tabular}{lll}
\hline Chlorophyll fluorescence kinetic parameters & W16-13 & WY16-13 \\
\hline$F_{0}$ & $4359.42 \pm 112.49^{* *}$ & $3079.71 \pm 337.95$ \\
$F_{m}$ & $30,025.85 \pm 845.46^{* *}$ & $23,454.71 \pm 2585.67$ \\
$F_{v} / F_{m}$ & $0.85 \pm 0.001$ & $0.87 \pm 0.003^{* *}$ \\
$A B S / R C$ & $0.81 \pm 0.04^{* *}$ & $0.69 \pm 0.03$ \\
$D I_{o} / R C$ & $0.12 \pm 0.006^{* *}$ & $0.09 \pm 0.006$ \\
$V_{j}$ & $0.29 \pm 0.02$ & $0.31 \pm 0.02$ \\
$P l_{\text {abs }}$ & $16.38 \pm 2.58$ & $22.46 \pm 4.01^{* *}$ \\
$P I_{\text {total }}$ & $13.61 \pm 2.74$ & $34.62 \pm 3.91^{* *}$ \\
\hline
\end{tabular}

Error bars indicate means \pm SD based on three independent experiments. Significant differences were determined using the Student's $t$ test in WY16-13 compared with W16-13 $(* * 0<0.01)$ 

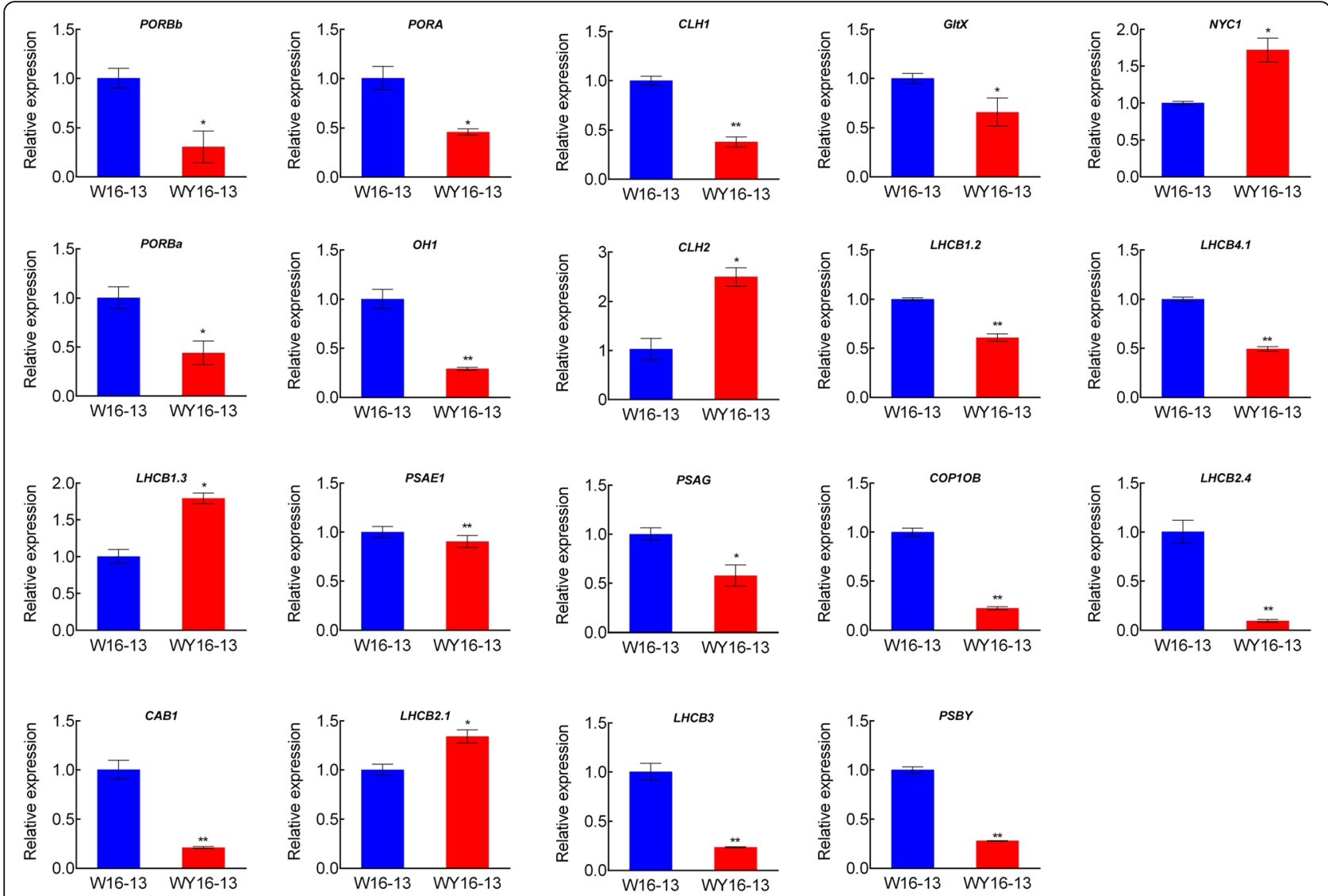

Fig. 10 qRT-PCR of 19 DEGs in "WY16-13" and "W16-13" leaves. Error bars indicate means \pm SD based on three independent experiments. Significant differences were determined using the Student's $t$ test in WY16-13 compared with W16-13( $\left(^{*} p<0.05,{ }^{* *} p<0.01\right)$

which encodes heme oxygenase, caused heme accumulation and resulted in blockage of Chl synthesis. The results in this study provide molecular evidence for the development of the yellow-green leaf phenotype as well as insights into using the yellow leaf trait as marker for breeding.

\section{Methods}

\section{Plant materials}

The green leaf Brassica campestris L. cultivar (wild-type, W16-13) and the yellow-green leaf B. campestris L. cultivar (mutant, WY16-13) were used in the present study. The experiment was conducted in the breeding basin in Hefei, Anhui Province, China (north latitude 31.86, east longitude 117.26). Seedlings were planted in the growth chamber of $24 \pm 1{ }^{\circ} \mathrm{C}$ (day) and $16 \pm 1{ }^{\circ} \mathrm{C}$ (night) with a relative humidity of $75-80 \%$ and the light intensity was $300 \mu \mathrm{mol} \cdot \mathrm{m}^{-2} \cdot \mathrm{s}^{-1}$ in a $14-\mathrm{h} / 10-\mathrm{h}$ light/dark photoperiod. After 20 days of transplanting, the third fully unfolded leaves from the center were collected for determination of photosynthetic efficiency and assessment of chloroplast ultrastructure. The samples were immediately frozen in liquid nitrogen after collection and stored at $-80^{\circ} \mathrm{C}$ for physiological and biochemical experiments.

\section{Morphological observation}

Morphological observation was performed every five days after planting. Leaf color values were estimated using a Chroma meter (CR-400-C, Konica Minolta Sensing Americas, Inc., Ramsey, NJ, USA) on the upper surface of the third unfolded leaf. A total of 10 plants were measured, and the two phenotypes were repeated thrice at the same leaf position. Plant height and expansion were measured with a rectilinear scale. Twenty WY16-13 and W16-13 plants with consistent growth and robust growth were measured as replicates.

\section{Measurement of $\mathrm{Chl}$ metabolite content}

Chl was extracted with a mixture containing acetone, ethanol, and water (4.5:4.5:1, volume ratio) according to Strain et al., with minor modifications [48]. Fresh leaf $(0.2 \mathrm{~g})$ was extracted in the dark for $26 \mathrm{~h}$, and the absorbance was determined by UV-vis spectrophotometer 
(TU1950, PERSEE, Beijing, China) at wavelengths of 665, 649, and $470 \mathrm{~nm}$.

Glu content was estimated using a Solarbio reagent kit (Cat \#BC1580, Beijing Solarbio Science \& Technology Co., Ltd., China). The content of ALA was measured as described by Mauzerall et al. [49]. PBG, Urogen III, and Coprogen III contents were measured according to Bogorad [50]. Proto IX, Mg-Proto IX, and Pchlide contents were measured according to Hodgins et al. [51].

\section{Measurement of $\mathrm{Chl}$ a fluorescence transients}

The fluorescence parameters were measured using a continuous excitation fluorometer Pocket Plant Efficiency Analyzer (PEA, Hansatech, UK). The initial fluorescence $F_{0}$ and the maximal fluorescence $F_{m}$, which respectively represented different fluorescence yields as the reaction center of PSII is fully opened or closed, were measured after the third functional leaves of the plants adapting in the dark for about $30 \mathrm{~min}$. A fast Chl fluorescence induction curve was analyzed with Biolyzer 3.0 software (Bioenergetics Lab., Geneva, Switzerland).

\section{Transmission electron microscopy}

Fresh functional leaf sections $(\leq 2 \mathrm{~mm} \times 5 \mathrm{~mm})$ were fixed with $2.5 \%$ glutaraldehyde in $0.1 \mathrm{M}$ PBS (sodium phosphate buffer, $\mathrm{pH} 7.0$ ) for $12 \mathrm{~h}$ at $4{ }^{\circ} \mathrm{C}$, and then washed thrice with $0.1 \mathrm{M}$ PBS (pH 7.0). The samples were post fixed with osmium tetroxide $(1 \%, \mathrm{w} / \mathrm{v})$ for $2 \mathrm{~h}$ and again washed thrice by $0.1 \mathrm{M}$ PBS ( $\mathrm{pH} 7.0$ ). Samples were dehydrated across an alcohol gradient (30, 50, 70, 80, 90, and 95\%) for $15 \mathrm{~min}$ at each concentration, then treated with absolute ethanol for $20 \mathrm{~min}$, and finally transferred to absolute acetone for $20 \mathrm{~min}$. The samples were infiltrated and embedded in Spurr's epoxy resin. After staining with uranyl acetate followed by lead citrate, the tissues were observed on a transmission electron microscope (HT-7700, Hitachi, Tokyo, Japan) at an accelerating voltage of $80.0 \mathrm{kV}$.

\section{RNA extraction, library construction, and RNA-seq}

Total RNA was extracted from the W16-13 and WY1613 leaf samples using a mirVana miRNA isolation kit (Ambion, TX, USA) according to the manufacturer's instructions. The integrity of extracted RNA was assessed using an Agilent 2100 Bioanalyzer (Agilent Technologies, Santa Clara, CA, USA). Sequencing libraries were prepared using TruSeq Stranded mRNA LT Sample Prep Kit (Illumina, San Diego, CA, USA) following the manufacturer's instructions. Then, these libraries were sequenced on an Illumina HiSeq 2500 platform (Biomarker Biotech, Beijing, China).

\section{Quality control and mapping of reads}

The transcriptome sequencing and analysis were conducted by OE Biotech Co., Ltd. (Shanghai, China). The raw reads were processed to remove low-quality reads, adapters, and reads with poly-A or ploy- $\mathrm{N}$ to obtain clean reads using Trimmomatic (version 0.36) [52]. Then, clean reads were mapped to the Brassica rapa reference genome (http:// brassicadb.org/brad/) using hisat2 (version 2.2.1.0) [53].

\section{Gene expression analysis}

Gene expression level analysis was performed using DESeq (version 1.18.0) [54] R package, and genes were normalized with FPKM [55] using cufflinks (version 2.2.1) [56]. Genes with $p$-value $<0.05$ and fold change $>2$ or $<0.5$ were considered DEGs between samples. Hierarchical cluster analysis of DEGs was performed to assess genes expression pattern. GO enrichment analysis of DEGs was performed using the GOseq $R$ package. The enrichment of the DEGs in KEGG pathways was assessed using KOBAS software [57, 58].

\section{Validation of DEGs by qRT-PCR}

Accuracy of transcriptome sequencing data was validated by qRT-PCR. Total RNA was extracted from leaves using a plant RNA extraction kit (Takara Biomedical Technology Co., Beijing, China). First-strand cDNA was synthesized using the PrimeScript ${ }^{\mathrm{Tm}}$ RT reagent kit (TaKaRa). qRT-PCR was performed in a $20-\mu \mathrm{L}$ reaction volume containing $1 \mu \mathrm{L}$ of $100 \mathrm{ng}$ cDNA, $1 \mu \mathrm{L}$ each of $10 \mu \mathrm{M}$ forward and reverse primers, $10 \mu \mathrm{L}$ SYBR Premix Ex Taq II (Takara), and filled with $7 \mu \mathrm{L} \mathrm{dd}_{2} \mathrm{O}$. Gene-specific primers were designed using Primer Software version 5.0 (Premier Biosoft International, CA, USA). The primers used for qRT-PCR are listed in Additional file 7: Table S4. BnaActin gene was used as reference [21]. Relative expression levels were calculated as $2^{-\Delta \Delta C T}[59]$.

\section{Statistical analysis}

Data were expressed as the mean \pm SD with three biological replicates. All of the data was subjected to an analysis of variance (ANOVA). The mean separation was performed using the Student's $t$ test with a significance level of $p<0.05$. Analyses were conducted using SPSS v22.0 for Windows (SPSS Inc., USA). The related figures were drawn using GraphPad Prism v6.0 (http://www. graphpad.com/scientific-software/prism/) and Origin Pro v9.1 software (OriginLab Corp., MA, USA).

\section{Abbreviations}

Chl: Chlorophyll; DEGs: Differentially expressed genes; GO: Gene Ontology; KEGG: Kyoto Encyclopedia of Genes and Genomes; Car: Carotenoid; Glu: Glutamate; ALA: 5-aminolevulinic acid; PBG: Porphobilinogen; Urogen III: Uroporphyrinogen III; Coprogen III: Coproporphyrinogen III; Proto IX: Protoporphyrin IX; Mg-Proto IX: Mg-protoporphyrin IX:

Pchlide: Protochlorophyllide; qRT-PCR: Quantitative real-time polymerase chain reaction; LHC: Light-harvesting chlorophyll protein complex; PSII: Photosystem II; PSI: Photosystem I 


\section{Supplementary Information}

The online version contains supplementary material available at https://doi. org/10.1186/s12864-021-07573-7.

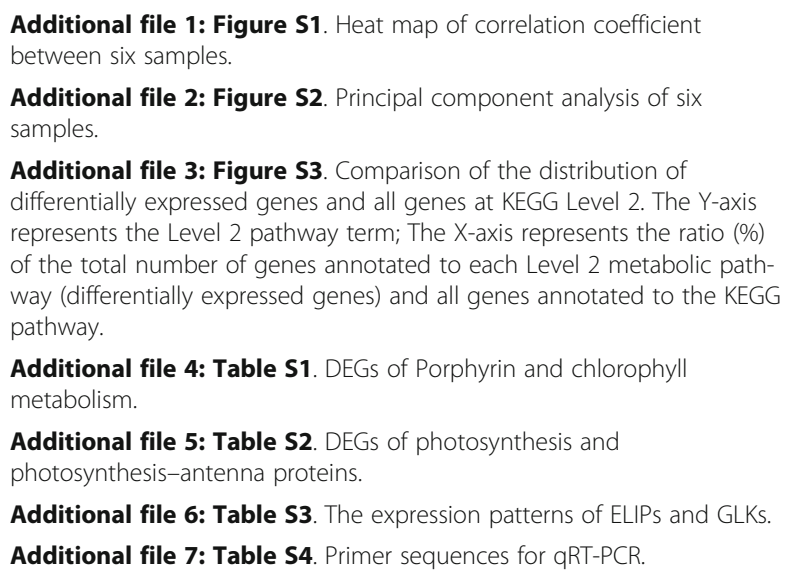

Additional file 4: Table S1. DEGs of Porphyrin and chlorophyll metabolism.

Additional file 5: Table S2. DEGs of photosynthesis and

photosynthesis-antenna proteins.

Additional file 6: Table S3. The expression patterns of ELIPS and GLKs.

Additional file 7: Table S4. Primer sequences for qRT-PCR.

\section{Acknowledgements}

We thank LetPub (www.letpub.com) for its linguistic assistance during the preparation of this manuscript.

\section{Authors' contributions}

LN conducted experiments and wrote the manuscript. LY and CW directed the study, including experimental design and manuscript revision. $Y Z, L Z$ and YW analyzed the data. SZ, JH, GC and XT supervised the study. All authors have read and approved the final manuscript.

\section{Funding}

This work was supported by the Natural Science Research Projects of Anhui Universities (KJ2020ZD12), the National Natural Science Foundation of China (No. 31701910), the Graduate Innovation Fund of Anhui Agricultural University (2020ysj-13), the Academic and Technical Leaders Reserve Candidate Project of Anhui Province, China (2018H181), and the Germplasm Resource Nursery of Wucai (201901n06030006).

\section{Availability of data and materials}

The dataset supporting the conclusions of this article is available in the NCBI's BioProject database [PRJNA683756].

\section{Declarations}

\section{Ethics approval and consent to participate}

The plants of wucai (Brassica campestris L.) were grown in the greenhouse of Anhui Agricultural University for sample collection. The research conducted in this study neither required approval from an ethics committee, nor involved any human or animal subjects. No specific permits were required for the described field studies. The location is not privately-owned or protected in any way, and the field studies did not involve endangered or protected species. We complied with the IUCN Policy Statement on Research Involving Species at Risk of Extinction and the Convention on the Trade in Endangered Species of Wild Fauna and Flora.

\section{Consent for publication}

Not applicable.

\section{Competing interests}

The authors declare that they have no competing interests.

\section{Author details}

${ }^{1}$ College of Horticulture, Vegetable Genetics and Breeding Laboratory, Anhui Agricultural University, 130 West Changjiang Road, Hefei 230036, Anhui, China. ${ }^{2}$ Provincial Engineering Laboratory for Horticultural Crop Breeding of Anhui, 130 West of Changjiang Road, Hefei 230036, Anhui, China. ${ }^{3}$ Wanjiang Vegetable Industrial Technology Institute, Maanshan 238200, Anhui, China.
Received: 13 December 2020 Accepted: 25 March 2021 Published online: 12 April 2021

\section{References}

1. Fromme P, Melkozernov A, Jordan P, Krauss N. Structure and function of photosystem I: interaction with its soluble electron carriers and external antenna systems. FEBS Lett. 2003;555(1):40-4. https://doi.org/10.1016/S00145793(03)01124-4.

2. Eckhardt U, Grimm B, Hortensteiner S. Recent advances in chlorophyll biosynthesis and breakdown in higher plants. Plant Mol Biol. 2004;56(1):1-14.

3. Wang P, Gao J, Wan C, Zhang F, Xu Z, Huang X, et al. Divinyl chlorophyll (ide) a can be converted to Monovinyl chlorophyll (ide) a by a Divinyl Reductase in Rice. Plant Physiol. 2010;153(3):994-1003. https://doi.org/10.11 04/pp.110.158477.

4. Liu W, Fu Y, Hu G, Si H, Zhu L, Wu C, et al. Identification and fine mapping of a thermo-sensitive chlorophyll deficient mutant in rice (Oryza sativa L.). Planta. 2007;226(3):785-95. https://doi.org/10.1007/s00425-007-0525-Z.

5. Sakuraba Y, Rahman ML, Cho S-H, Kim Y-S, Koh H-J, Yoo S-C, et al. The rice faded green leaf locus encodes protochlorophyllide oxidoreductaseB and is essential for chlorophyll synthesis under high light conditions. Plant J. 2013; 74(1):122-33. https://doi.org/10.1111/tpj.12110.

6. Sakuraba Y, Park S-Y, Kim Y-S, Wang S-H, Yoo S-C, Hoertensteiner S, et al. Arabidopsis STAY-GREEN2 is a negative regulator of chlorophyll degradation during leaf senescence. Mol Plant. 2014;7(8):1288-302. https://doi.org/10.1 093/mp/ssu045.

7. Zhang K, Liu Z, Shan X, Li C, Tang X, Chi M, et al. Physiological properties and chlorophyll biosynthesis in a Pak-choi (Brassica rapa L. ssp chinensis) yellow leaf mutant, pylm. Acta Physiol Plant. 2017;39(1):22.

8. Maekawa M, Hase Y, Shikazono N, Tanaka A. Induction of somatic instability in stable yellow leaf mutant of rice by ion beam irradiation. Nuclear Instruments \& Methods in Physics Research Section B-Beam Interactions with Materials and Atoms. 2003;206:579-85.

9. Dan-Xia W, Rui-Dang Q, Rong-Feng HJJA. Technology: map-based cloning and function analysis of Rice yellow leaf mutant yl1; 2015.

10. Chao WU, Ya-Ping FU, Guo-Cheng HU, Hua-Min SI, Xu-Ri L, Zong-Xiu S, et al. Identification and Fine Mapping of a Spotted and Yellow Leaf Mutant in Rice; 2011.

11. Wang P, Li C, Wang Y, Huang R, Sun C, Xu Z, Zhu J, Gao X, Deng X, Wang PJS: Identification of a Geranylgeranyl reductase gene for chlorophyll synthesis in rice 2014, 3(1):201.

12. Wu Z, Zhang X, He B, Diao L, Wan JJPP: A Chlorophyll-Deficient Rice Mutant with Impaired Chlorophyllide Esterification in Chlorophyll Biosynthesis 2007, 145(1):29-40.

13. Luo T, Luo S, Araujo WL, Schlicke H, Rothbart M, Yu J, et al. Virus-induced gene silencing of pea CHLI and CHLD affects tetrapyrrole biosynthesis, chloroplast development and the primary metabolic network. Plant Physiol Biochem. 2013;65:17-26. https://doi.org/10.1016/j.plaphy.2013.01.006.

14. Yang Y-L, Xu J, Rao Y-C, Zeng Y-J, Liu H-J, Zheng T-T, et al. Cloning and functional analysis of pale-green leaf (PGL10) in rice (Oryza sativa L.). Plant Growth Regul. 2016;78(1):69-77. https://doi.org/10.1007/s10725-015-0075-5.

15. Park S-Y, Yu J-W, Park J-S, Li J, Yoo S-C, Lee N-Y, et al. The senescenceinduced staygreen protein regulates chlorophyll degradation. Plant Cell. 2007;19(5):1649-64. https://doi.org/10.1105/tpc.106.044891.

16. Tzvetkova-Chevolleau T, Franck F, Alawady AE, Dall'Osto L, Carriere F, Bassi $\mathrm{R}$, et al. The light stress-induced protein ELIP2 is a regulator of chlorophyll synthesis in Arabidopsis thaliana. Plant J. 2007:50(5):795-809. https://doi. org/10.1111/j.1365-313X.2007.03090.x.

17. Hu H, Wang L, Wang Q, Jiao L, Hua W, Zhou Q, et al. Photosynthesis, chlorophyll fluorescence characteristics, and chlorophyll content of soybean SEEDLINGS under combined stress of BISPHENOL a and cadmium. Environ Toxicol Chem. 2014;33(11):2455-62. https://doi.org/10.1002/etc.2720.

18. Lee S-K, Jeon J-S, Boernke F, Voll L, Cho J-I, Goh C-H, et al. Loss of cytosolic fructose-1,6-bisphosphatase limits photosynthetic sucrose synthesis and causes severe growth retardations in rice (Oryza sativa). Plant Cell Environ. 2008;31(12):1851-63. https://doi.org/10.1111/j.1365-3040.2008.01890.x.

19. Albrecht V, Simkova K, Carrie C, Delannoy E, Giraud E, Whelan J, Small ID, Apel K, Badger MR, Pogson BJJPC: The Cytoskeleton and the PeroxisomalTargeted SNOWY COTYLEDON3 Protein Are Required for Chloroplast Development in Arabidopsis 2010, 22(10):3423-3438.

20. Zhao M, Yuan L, Wang J, Xie S, Zheng Y, Nie L, et al. Transcriptome analysis reveals a positive effect of brassinosteroids on the photosynthetic capacity 
of wucai under low temperature. BMC Genomics. 2019;20(1):810. https://doi. org/10.1186/s12864-019-6191-2.

21. Xie S, Nie L, Zheng Y, Wang J, Zhao M, Zhu S, et al. Comparative proteomic analysis reveals that chlorophyll metabolism contributes to leaf color changes in Wucai ( Brassica campestris L.) responding to cold acclimation. J Proteome Res. 2019;18(6):2478-92. https://doi.org/10.1021/acs.jproteome. $9 \mathrm{~b} 00016$.

22. Zhu XW, Pan Y, Liu Z, Liu YC, Zhong DY, Duan ZB, et al. Mutation of YL results in a yellow leaf with chloroplast RNA editing defect in soybean. Int J Mol Sci. 2020;21(12):15

23. Li C, Ma FR, Jiao RJ, Chen CP, Wang Q, Xiao FL, et al. Mutation in mgProtoporphyrin IX Monomethyl Ester Cyclase causes yellow and spotted leaf phenotype in Rice. Plant Mol Biol Rep. 2019;37(4):253-64. https://doi.org/1 0.1007/s11105-019-01152-7.

24. Miao $H$, Zhang $S$, Wang $M$, Wang $Y$, Weng $Y$, Gu X. Fine Mapping of Virescent Leaf Gene v-1 in Cucumber (Cucumis sativus L.). Int J Mol Sci. 2016;17(10):1602.

25. Azarin K, Usatov A, Makarenko M, Kozel N, Kovalevich A, Dremuk I, et al. A point mutation in the photosystem I P700 chlorophyll a apoprotein A1 gene confers variegation inHelianthus annuusL. Plant MolBiol. 2020;103(45):373-89.

26. Hu X, Makita S, Schelbert S, Sano S, Ochiai M, Tsuchiya T, et al. Reexamination of Chlorophyllase function implies its involvement in defense against chewing herbivores. Plant Physiol. 2015;167(3):660-70 https://doi.org/10.1104/pp.114.252023.

27. Cheng Y, Dong Y, Yan H, Ge W, Shen C, Guan J, et al. Effects of 1-MCP on chlorophyll degradation pathway-associated genes expression and chloroplast ultrastructure during the peel yellowing of Chinese pear fruits in storage. Food Chem. 2012;135(2):415-22. https://doi.org/10.1016/j. foodchem.2012.05.017.

28. Kusaba M, Ito H, Morita R, lida S, Sato Y, Fujimoto M, et al. Rice NONYELLOW COLORING1 is involved in light-harvesting complex II and grana degradation during leaf senescence. Plant Cell. 2007;19(4):1362-75. https:// doi.org/10.1105/tpc.106.042911

29. Bakhsh A, Kim SH, Kim SW, Lim G-H, Lyu JI, Choi H-I, et al. Transcriptome analysis to identify candidate genes associated with the yellow-leaf phenotype of a Cymbidium mutant generated by $\gamma$-irradiation. PLoS One. 2020;15(1):e0228078.

30. Zhu L, Yang Z, Zeng X, Gao J, Liu J, Yi B, et al. Heme oxygenase 1 defects lead to reduced chlorophyll in Brassica napus. Plant Mol Biol. 2017;93(6): 579-92. https://doi.org/10.1007/s11103-017-0583-y.

31. Emborg TJ, Walker JM, Noh B, Vierstra RD. Multiple heme oxygenase family members contribute to the biosynthesis of the phytochrome chromophore in Arabidopsis. Plant Physiol. 2006;140(3):856-68. https://doi.org/10.1104/ pp.105.074211.

32. Li Q, Zhu F-Y, Gao X, Sun Y, Li S, Tao Y, et al. Young leaf Chlorosis 2 encodes the stroma-localized heme oxygenase 2 which is required for normal tetrapyrrole biosynthesis in rice. Planta. 2014;240(4):701-12. https:// doi.org/10.1007/s00425-014-2116-0.

33. Chen H, Cheng ZJ, Ma XD, Wu H, Liu YL, Zhou KN, et al. A knockdown mutation of YELLOW-GREEN LEAF2 blocks chlorophyll biosynthesis in rice. Plant Cell Rep. 2013;32(12):1855-67. https:/doi.org/10.1007/s00299-013-1498-y.

34. Davis SJ, Kurepa J, Vierstra RD. The Arabidopsis thaliana HY1 locus, required for phytochrome-chromophore biosynthesis, encodes a protein related to heme oxygenases. Proc Natl Acad Sci U S A. 1999;96(11):6541-6. https://doi. org/10.1073/pnas.96.11.6541.

35. Allen JF, de Paula WB, Puthiyaveetil S, Nield J. A structural phylogenetic map for chloroplast photosynthesis. Trends Plant Sci. 2011;16(12):645-55. https://doi.org/10.1016/j.tplants.2011.10.004.

36. Bashir H, Qureshi MI, Ibrahim MM, labal M. Chloroplast and photosystems: impact of cadmium and iron deficiency. Photosynthetica. 2015;53(3):321-35. https://doi.org/10.1007/s11099-015-0152-z.

37. Naested $H$, Holm A, Jenkins $T$, Nielsen $H B$, Harris CA, Beale MH, et al. Arabidopsis VARIEGATED 3 encodes a chloroplast-targeted, zinc-finger protein required for chloroplast and palisade cell development. J Cell Sci. 2004;117(Pt 20):4807-18. https://doi.org/10.1242/jcs.01360.

38. Martin W, Rujan T, Richly E, Hansen A, Cornelsen S, Lins T, et al. Evolutionary analysis of Arabidopsis, cyanobacterial, and chloroplast genomes reveals plastid phylogeny and thousands of cyanobacterial genes in the nucleus. Proc Natl Acad Sci U S A. 2002;99(19):12246-51. https://doi.org/10.1073/pna S.182432999.
39. Zeng O, Chen XB, Wood AJ. Two early light-indudible protein (ELIP) CDNAs from the resurrection plant Tortula ruralis are differentially expressed in response to desiccation, rehydration, salinity, and high light. J Exp Bot. 2002; 53(371):1197-205. https://doi.org/10.1093/jexbot/53.371.1197.

40. Hutin C, Nussaume L, Moise N, Moya I, Kloppstech K, Havaux M. Early lightinduced proteins protect arabidopsis from photooxidative stress. Proc Natl Acad Sci U S A. 2003;100(8):4921-6. https://doi.org/10.1073/pnas.0736939100.

41. Beck J, Lohscheider JN, Albert S, Andersson U, Mendgen KW, Rojas-Stuetz $M C$, et al. Small one-helix proteins are essential for photosynthesis in Arabidopsis. Front Plant Sci. 2017;8. https://doi.org/10.3389/fpls.2017.00007.

42. Heddad M, Noren H, Reiser V, Dunaeva M, Andersson B, Adamska I. Differential expression and localization of early light-induced proteins in Arabidopsis. Plant Physiol. 2006;142(1):75-87. https://doi.org/10.1104/pp.106. 081489

43. Zubo YO, Blakley IC, Franco-Zorrilla JM, Yamburenko MV, Solano R, Kieber JJ, et al. Coordination of chloroplast development through the action of the GNC and GLK transcription factor families. Plant Physiol. 2018;178(1):130-47. https://doi.org/10.1104/pp.18.00414.

44. Bastakis E, Hedtke B, Klermund C, Grimm B, Schwechheimer C. LLM-domain B-GATA transcription factors play multifaceted roles in controlling greening in Arabidopsis. Plant Cell. 2018;30(3):582-99. https://doi.org/10.1105/tpc.17.00947.

45. Waters MT, Wang P, Korkaric M, Capper RG, Saunders NJ, Langdale JA. GLK transcription factors coordinate expression of the photosynthetic apparatus in Arabidopsis. Plant Cell. 2009;21(4):1109-28. https://doi.org/10.1105/tpc.1 08.065250.

46. Fitter DW, Martin DJ, Copley MJ, Scotland RW, Langdale JA. GLK gene pairs regulate chloroplast development in diverse plant species. Plant J. 2002; 31(6):713-27. https://doi.org/10.1046/j.1365-313X.2002.01390.x.

47. Waters MT, Moylan EC, Langdale JA. GLK transcription factors regulate chloroplast development in a cell-autonomous manner. Plant J. 2008;56(3): 432-44. https://doi.org/10.1111/j.1365-313X.2008.03616.x.

48. Strain HH, Svec WAJC. Extraction, Separation, Estimation, and Isolation of the Chlorophylls *; 1966. p. 21-66.

49. Mauzerall $D$, Granick $S$. The occurrence and determination of delta-aminolevulinic acid and porphobilinogen in urine. J Biol Chem. 1956;219(1):43546. https://doi.org/10.1016/50021-9258(18)65809-0.

50. Bogorad L. Porphyrin synthesis. Methods Enzymol. 1962:5:885-95. https:// doi.org/10.1016/S0076-6879(62)05334-3.

51. Hodgins RR, Van Huystee RB. Rapid simultaneous estimation of Protoporphyrin and mg-Porphyrins in higher plants. J Plant Physiol. 1986; 125(3-4):311-23. https://doi.org/10.1016/50176-1617(86)80153-5.

52. Bolger AM, Lohse $M$, Usadel B. Trimmomatic: a flexible trimmer for Illumina sequence data. Bioinformatics. 2014;30(15):2114-20. https://doi.org/10.1093/ bioinformatics/btu170

53. Kim D, Langmead B, Salzberg SL. HISAT: a fast spliced aligner with low memory requirements. Nat Methods. 2015;12(4):357-U121. https://doi.org/1 0.1038/nmeth.3317.

54. Anders SWH. Differential expression of RNA Seq data at the gene level the DESeq package. EMBL. 2013.

55. Roberts A, Trapnell C, Donaghey J, Rinn JL, Pachter L. Improving RNA-Seq expression estimates by correcting for fragment bias. Genome Biol. 2011; 12(3):R22.

56. Trapnell C, Williams BA, Pertea G, Mortazavi A, Kwan G, van Baren MJ, et al. Transcript assembly and quantification by RNA-Seq reveals unannotated transcripts and isoform switching during cell differentiation. Nat Biotechnol. 2010;28(5):511-U174. https://doi.org/10.1038/nbt.1621.

57. Kanehisa M, Araki M, Goto S, Hattori M, Hirakawa M, Itoh M, et al. KEGG for linking genomes to life and the environment. Nucleic Acids Res. 2008;36:D480-4.

58. Altermann E, Klaenhammer TR. PathwayVoyager: pathway mapping using the Kyoto encyclopedia of genes and genomes (KEGG) database. BMC Genomics. 2005;6(1). https://doi.org/10.1186/1471-2164-6-60.

59. Schmittgen $T D$, Livak KJ. Analyzing real-time PCR data by the comparative $C(T)$ method. Nat Protoc. 2008;3(6):1101-8. https://doi.org/10.1038/nprot.2008.73.

\section{Publisher's Note}

Springer Nature remains neutral with regard to jurisdictional claims in published maps and institutional affiliations. 